\title{
Acciones creativas como factor clave del perfil emprendedor del estudiante universitario
}

\section{Creative actions as a key factor in the entrepreneurial profile of the university student}

\author{
PACHECO, Carlos M. ${ }^{1}$ \\ NIEBLES, William A. ${ }^{2}$ \\ HERNÁNDEZ, Hugo G. ${ }^{3}$
}

\begin{abstract}
Resumen
La investigación busca identificar las acciones creativas como factor clave del perfil emprendedor del estudiante. La misma fue de tipo descriptivo, no experimental, transeccional y de campo. La población fue de 54 docentes. Se utilizó un cuestionario con 28 items, cuya confiabilidad fue 0,91. Los datos se analizaron bajo estadística descriptiva estimándose las medidas de tendencia centra, así como las medidas de dispersión. Los hallazgos consideran que los estudiantes desarrollan acciones creativas que fomentan y refuerzan su perfil emprendedor.

Palabras clave: acción creativa, innovación, visión de futuro, iniciativa

Abstract

The research seeks to identify creative actions as a key factor in the student's entrepreneurial profile. It was descriptive, not experimental, transectional and field. The population was (54) teachers. A questionnaire was used with 28 items, with a value of 0.91 of reliability. The data were analyzed under descriptive statistics estimating the focus trend measures as well as the dispersion measures. The findings consider that students develop creative actions that encourage and reinforce their entrepreneurial profile.

keywords: creative action, innovation, vision of the future, initiative
\end{abstract}

\section{Introducción}

En la actualidad los sistemas de acreditación de programas e instituciones que se han desarrollado concretamente en el ámbito de la educación superior, han generado un alto nivel de competitividad enlazando diferentes tendencias hacia el desarrollo de procesos y habilidades que en siglos pasados eran algo indiferente como parte del proceso educativo. En este sentido, para el siglo XXI, las universidades se han visto en la necesidad de adecuar sus procesos para adaptarse a los nuevos eventos del contexto, para cumplir con su misión. En virtud

\footnotetext{
${ }^{1}$ Magister en Administración de Empresas, Especialista en Gerencia de Proyectos, Administrador de Empresas Docente Universidad de Sucre. carlos.pacheco@unisucre.edu.co

${ }^{2}$ Doctor en Ciencias Gerenciales, Magister en Dirección Estratégica, Administrador de Empresas. Docente de la Universidad de Sucre. william.niebles@unisucre.edu.co

${ }^{3}$ Magister en Sistema de Gestión, Especialista en Estudios Pedagógicos, Especialista en Diseño y Evaluación de proyectos, Ingeniero Industrial. Docente

Programa de Administración de Empresas Universidad del Atlántico-Colombia. hugoghernandezpalma@gmail.com
} 
de promover transformaciones que emergen desde la conceptualización en organizaciones que innovan y aprenden de sus propias experiencias como de su desempeño.

De acuerdo a lo expresado, se percibe que las instituciones de educación superior, han ido adaptándose a las exigencias de la sociedad, en virtud de promover profesionales destacados, eficientes y proactivos; y en su más importante acción, individuos emprendedores.

Al respecto, García et al. (2018) y Aristimuño, \& Guaita (2011), señalaron que el espacio universitario responde a la presión del entorno social y económico, lo que ha derivado en una demanda creciente de popularización e internacionalización de investigaciones como la educación; y de mayor calidad, que Los resultados deben ser cuantificables para aportar beneficios económicos a la sociedad, Valencia, Montoya y Montoya (2016) enfatizaron que han surgido las intenciones emprendedoras, y lo definen como una especie de atención humana enfocada en la consecución de metas. En este caso, la constitución de una empresa es un negocio que tiene un cierto impacto en las acciones que realizan los individuos para lograr los objetivos anteriores.

En este sentido, Jiménez, Hernández y Pitre (2018) confirmaron que el principal factor de competitividad organizacional radica en sus empleados. Esta flexibilidad en la gestión está relacionada con una serie de funciones que brindan a los emprendedores opciones para encontrar estrategias alternativas y dar respuesta a diversas demandas en un entorno competitivo altamente dinámico (Durán, Parra y Márceles, 2015).

Esta situación implica un proceso de adaptación rápida y activa de las instituciones educativas al entorno dinámico, lo que exige a los estudiantes forjar su capacidad emprendedora, con visión de futuro, desarrollando sus percepciones, habilidades y competencias relacionadas con el proceso de hacer frente a los cambios, según Parra et al (2019), produce una gran sensibilidad frente a las transformaciones internas del entorno, como su agilidad para reconfigurar las actividades y acciones creativas para afrontar las condiciones que surgen de tales situaciones.

Cabe señalar que el entorno universitario enfrenta retos de futuro, por lo que debe ser coherente con los constantes cambios que genera la dinámica social y educativa de cada país, especialmente la situación en Colombia. Sánchez et al. (2019) y Hernández \& Hernández (2008) se basan en innovadoras prácticas de enseñanza, gestión y técnicas, y se comentan los esfuerzos por estimular los talentos humanos, las habilidades cognitivas y mejorar las habilidades como parte de la creatividad, siempre teniendo en cuenta que la democracia, orientada a las personas, es la fuerza impulsora del cambio para su sociedad. De allí, que las universidades tienen un papel importante en la configuración de la sociedad dada su alta responsabilidad en la formación de la humanidad como en la generación del conocimiento científico (Viloria, Rodriguez et al., 2019).

Estas exigencias provocadas por esta nueva sociedad del conocimiento ha tocado a los entornos educativos como a todos sus miembros en el mundo, específicamente el area de las tecnologías de información y comunicación como lo plantean Viloria, Crissien et al (2019), vinculando las TIC como impulsor fundamental de los procesos continuos de cambio, lo que además ha ocasionado la necesidad de formularse una nueva visión, sobre el rol del profesor, que según Pérez et al (2019), determinan como los retos a asumir, de igual manera las universidades en el siglo XXI.

Sustentado en lo expuesto anteriormente, puede aseverarse que en las universidades se requiere de un sistema de gestión integral que vincule las posibilidades de promover emprendedores, con habilidades y competencias que contribuyan al crecimiento de las personas, Hernández, Parra, Durán et al (2018), así como que estos estudiantes sean autodeterminados, con mayor esperanza, para que alcancen mejores niveles de excelencia en su desempeño profesional. Moreno et al (2018) manifiestan que en este contexto deben ser más asertivos, con mayor manejo de la incertidumbre, permitiendo consolidar una autoestima basada en los resultados, es decir 
que contribuya a lo que se conoce como el desarrollo de las competencias básicas para el crecimiento ocupacional.

Atendiendo a estas consideraciones, desde las instituciones de educación superior orientadas hoy en día al desarrollo de procesos internos que permitan dar respuesta al entorno, se han planteado propuestas y estrategias para que surjan, desde las aulas, profesionales adaptados a esa necesidad global, entre ellas la de acciones creativas arraigadas al perfil del emprendedor.

Dichas instituciones están mediadas por una cultura ejercida en ella, procedimientos que podrían limitar la identidad ideológica corporativa, flexibles y abiertos a los cambios, reconociendo la necesidad de cambiar, adaptarse y promover emprendedores e innovadores; permitiendo así fortalecer el desarrollo profesional, para lo que se destacan las universidades de la costa caribe Colombiana, entre ellas la universidad de Sucre y la universidad del Atlántico.

Se ha determinado que estas instituciones de educación superior (IES), requieren adoptar enfoques y procesos que les permitan mejorar de forma continua el producto formado y constituir a la innovación como parte del proceso continuo, por talrazón, se deben adaptar los diseños organizacionales en función de los cambios y de acuerdo con el entorno, según Rivera et al (2019), también promover conocimientos en el docente.

Indudablemente que este nuevo desafío de las IES, esboza un escenario que requiere de un compromiso claro, con acciones que promuevan generar y promover el conocimiento creativo, en función de alcanzar una sociedad más justa y próspera, siendo el punto de partida para que el estudiante emprendedor pueda crear spin-off, definido por Zamfir, et al (2013) la manera eficaz para transferir conocimiento y tecnología desde la investigación universitaria u otra organización hacia la economía.

Esta concepción, incluye un amplio marco de actividades teniendo en consideración tanto los costos como los beneficios como parte del sistema de innovación en su conjunto lo que hace posible una efectiva caracterización del perfil emprendedor como parte de su acción creativa, aunado a la capacidad de participar en diferentes actividades que consagran las IES nacionales en diferentes sectores y areas.

En este orden de ideas, las universidades publicas (Universidad de Sucre y Universidad del Atlántico), han asumido la responsabilidad de las prácticas en educación superior, para desarrollar y potenciar el perfil emprendedor del estudiante de tal manera de responder a las necesidades emergentes que requieren de procesos de generación y transferencia de conocimiento, a través del trabajo colaborativo.

De lo antes expuesto, surgió la inquietud de realizar un estudio que se oriente a determinar las acciones creativas como factor clave del perfil emprendedor del estudiante Universitario.

\subsection{Perfil del emprendedor}

Como señala Sánchez (2008), la creciente demanda de instituciones y organizaciones representativas del sector social y comercial, público y privado en torno a la universidad ha imposibilitado la satisfacción de las necesidades sociales de la facultad, lo que demuestra el papel tradicional del formador profesional en el mercado laboral. Por tanto, los emprendedores tienen capacidad de creatividad e innovación, que es una de sus características.

En opinión de Pitre, Rodriguez, Hernández, \& Cardona (2017) el emprendimiento no solo debe concebirse en base a las características psicológicas y de comportamiento personales del emprendedor, ni debe ser concebido a partir del proceso que inicia el emprendedor para aprovechar las oportunidades que se presentan en el entorno, sino que debe ser considerado como un todo universal. La fusión de diversas disciplinas dio paso a un nuevo enfoque antropológico, ontológico y epistemológico que permitió la comprensión y desarrollo del comportamiento emprendedor. 
Por otro lado, el emprendedor debe ser consciente de que tiene una actitud y un talento ideales para "ganar dinero" y también debe ganar dinero (Jiménez, 2015). Del mismo modo, aunque nadie puede discutir sobre ninguna actividad empresarial legal, por su propia naturaleza, puede generar beneficios sociales porque puede crear puestos de trabajo y promover el desarrollo económico.

En este sentido, Connor (2009) y Gómez (2008) explica que convertirse en emprendedor no es solo un atributo profesional, es un comportamiento y una forma de existencia alcanzables, más que una cosa externa. Por eso, se considera al emprendedor como una persona que puede influir y apoyar a los demás para que logren sus objetivos con ilusión. Este elemento se relaciona lo dicho por Rivera y Santos (2015), quienes señalaron que el altruismo y la innovación juvenil son principalmente las motivaciones para estudiar este tipo de decisiones.

El autor menciona que los emprendedores son más proactivos en el ejercicio de sus capacidades de agencia que otros individuos, y la estructura social no impondrá condiciones, incluso si la estructura social lo afecta, como afectar sus oportunidades laborales desde los recursos (formación, financiación, etc.). Pero la la habilidad de creación de los emprendedores exige que el emprendor construya relaciones, especialmente si el objetivo es de carácter social, como el misionero (de manera Voluntaria) y la inserción en aspectos político.

De manera similar, Weitzel et al. (2010) citado en Rivera \& Santos (2015), argumentan que quienes tienen habilidades emprendedoras y quienes son más creativos proceden de una manera más generosa. A su vez, las teorías de estos autores muestran que el aumento de la capacidad personal y la participación tiene un efecto favorable en el desarrollo del altruismo y origina actitudes propicios para el emprendimiento. Además de lo anterior, Jaimes (2009) también describió tres aspectos relevantes de un emprendedor, como ser creativo, estar dispuesto a asumir riesgos y poseer habilidades comunicativas. Además, debe ser convincente en su decisión y abierto a resolver inuietudes, lo que implica una actitud orientada a aclarar, participar y compartir ideas. A continuación, se detalla la creatividad que caracteriza el perfil del emprendedor.

\subsection{Acciones creativas}

Sánchez (2008, p. 201) define la creatividad como "la capacidad de dar uso diferente a lo que ya se posee, de tal manera que se genere un impacto significativo", soportado en el deseo de superación e innovación sumado con la actitud positiva para lograr las metas propuestas. Se puede indicar que el espíritu creativo se puede entender como una forma de razonar y proceder enfocada en las oportunidades, pensado de forma global, con liderazgo equilibrado y riesgo planificado. Por otro lado, la creatividad está ligada a la habilidad de aprovechar todas las capacidades del individuo en cuanto a visualizar e imaginar los problemas como oportunidades para obtener la excelencia.

Para Camero (2008) es la habilidad vital con la cual se impulsa hacia la realización de grandes cosas, actuando de manera decidida, innovadora y motivada de tal manera que se cumplan objetivamente las metas propuestas y así poder alcanzar el éxito, convirtiendo los problemas organizacionales en oportunidades de mejora y superación.

En consecuencia supone desarrollar la iniciativa personal, la confianza en uno mismo, el dinamismo, el sentido crítico, el asumir riesgos, aspectos que conlleven a estar alerta ante las circunstancias que se le presentan, convirtiendo estas en grandes oportunidades. Igualmente la creatividad se considera una habilidad gerencial que permite percibir las oportunidades sociales con el fin de lograr sus objetivos, a través de una motivación constante y la habilidad de movilizar una serie de recursos en la oportunidad de materializarla en la obtención de la meta deseada hacia la creación de valor compartido.

El concepto de educación de la creatividad comienza con la proposición de que la creatividad está estrechamente relacionada con todas las áreas de la actividad humana y es producto de la evolución histórica. De este modo, 
creatividad significa buscar el cambio. Según Niebles et al. (2018), espertinente promover al menos la capacidad de afrontar cosas nuevas y dotarlas de creatividad a través de la libertad psicológica y un ambiente humanista profundo.

Según Gallagher y Gallagher (1994; citado por Williams, Ostwald, y Askland, 2010), explican que la creatividad es un proceso cognitivo que genera productos, desempeños o paradigmas nuevos o mejorados. Mostrando una similitud con otros autores, la inclinación de la investigación se basó en estos autores, dejando así una clara idea de creatividad; lo cual es una cualidad del pensamiento que permite al individuo generar muchas ideas, inventar nuevas ideas o combinar las existentes de manera novedosa.

Aunado a ello, Guilford (1977) citado en Rebollo \& Soubirón (2010) aporta que los profesores que estimulan el desarrollo creativo deben ser divergente en su pensamiento, lo que significa, utilizar los conocimientos pensando en nuevas maneras de realizar las cosas, apartir de las experiencias, para producir tantas soluciones como sea posible en cada situación que se presente. Los profesores más creativos serán aquellos que acepten ideas opuestas, preferiblemente, productos de su actuar docente. La labor docente deben estar orientada a estimular, apoyar y animar a sus alumnos a exponer sus pensamientos, siempre en función de resolver situaciones problemáticas, a partir de los temas de su interés, la clave está en plantear preguntas abiertas, promover el diálogo y Discutir las situaciones planteadas por los estudiantes, para fomentar independencia y actitud de liderazgo en su diario vivir .

El autor describe ciertas habilidades directamente vinculadas al pensamiento creativo que las cuales se muestran en el siguiente gráfico.

Gráfico 1

Acciones creativas

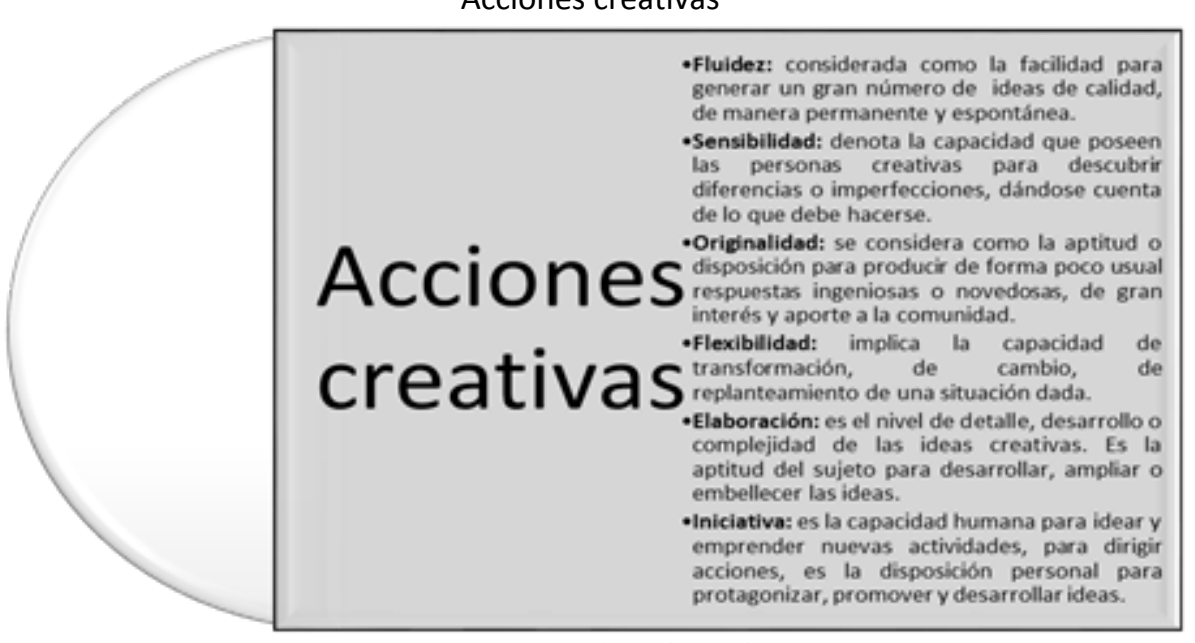

Fuente: Guilford (1977)

Por otra parte, conviene tener las características de los estudiantes posmodernos, definidos por la aseguridad en si mismo, la iniciativa, la libertad de pensamiento y de actuar, la constancia y la capacidad de resolución de problemas, aspectos que se restringen o no se fortalecen en la educación formal (Rebollo \& Soubirón, 2010).

Bajo este contexto, potenciar el pensamiento ceativo depende de la reflexión, el pensamiento flexible y divergente en los individuos, aplicados a la resolución de problemas, en lo posible con idependencia, para satisfacer con precisión, desde los propósitos formativos, las necesidades de la sociedad moderda, influenciada por el paradigma de la complejidad,

De acuerdo a lograr el perfil del emprendedor en las IES, se deben establecer diferentes actividades y orientaciones por parte del personal docente, en función de resaltar la creatividad, elemento pronóstico para 
futuros emprendedores, indudablemente se hace necesario que el joven exprese y ejecute sus pensamientos creativos, desenvuelva sus potencialidades innovadoras e intelectuales. En este caso, el proceso creativo es uno de los potenciales humanos más altos y complejos, lo que significa que la capacidad de pensar puede integrar procesos cognitivos menos complejos, incluso aquellos conocidos como procesos avanzados en cuanto a la realización de nuevas ideas o pensamientos.

\section{Metodología}

De acuerdo al método la investigación se orientó siguiendo los criterios del estudio cuantitativo, al pretender determinar las acciones creativas como factor clave del perfil emprendedor del estudiante universitario, dado que las variables pueden medirse numericamene a través de una ecuación que muestre sus variaciones Asimismo, es de tipo descriptiva, de campo, desarrolladas en la Universidad de Sucre y la Universidad del Atlántico, ubicadas en la costa caribe colombiana.

Además, se utilizó un diseño no experimental, por cuanto se estudió la creatividad como parte del perfil del emprendedor, determinando sus caractristicas fundamentales, sin ejercer influencia en el contexto, examinando desde su estado natural sin ninuna manipulación de variable, a partir de laa obserbavión tal y como ocurre en el contexto investigativo. Dentro de los diseños no experimentales, se clasificó como transeccional o transversal descriptiva.

En cuanto a la población, esta estuvo compuesta por 54 docentes de ambas instituciones pertenecientes a diferentes facultades de diferentes programas, y que tienen como similitud programas de emprendimiento. En consideración, la población de estudio poseen particularidades similares que requieren ser consideradas y representan la totalidad del fenómeno a analizar. para obtener los datos de la investigación.

Cuadro 1

Características de la Población

\begin{tabular}{ccc}
\hline Universidades & Facultades & Docentes \\
\hline Universidad de Sucre & 5 & 20 \\
Universidad del Atlántico & 10 & 34 \\
TOTAL & $\mathbf{1 5}$ & $\mathbf{5 4}$ \\
\hline
\end{tabular}

Fuente: Elaboración propia (2019)

Por otra parte, surge la técnica de recolección de datos, constituida por la técnica de la observación directa mediante la encuesta, cuyo contenido estuvo configurado por un conjunto de ítems, dirigidos a medir el nivel de información sobre las acciones creativas del perfil emprendedor del estudiante universitario. En este sentido, se construyó un instrumento con formato escala, constituido por 15 ítems, con Escala de Likert, tal y como se muestra en el siguiente cuadro.

Cuadro 2

Asignación de puntaje de respuesta

\begin{tabular}{|c|c|c|}
\hline Ítems & Ponderación & Respuestas \\
\hline \multirow{2}{*}{ Positivo } & 5 & Completamente de Acuerdo (CA) \\
\cline { 2 - 3 } & 4 & Medianamente de Acuerdo (MA) \\
\hline \multirow{2}{*}{ Neutro } & 3 & Ni de Acuerdo Ni en Desacuerdo (NAND) \\
\hline \multirow{2}{*}{ Rechazo } & 2 & Medianamente en Desacuerdo (MD) \\
\cline { 2 - 3 } & 1 & Completamente en Desacuerdo (CD) \\
\hline
\end{tabular}

Fuente: Elaboración propia (2019)

La escala está definida por grado o nivel y se considera apta para este trabajo, porque ayuda a distinguir el grado de comportamiento de los sujetos objeto de estudio. Asimismo, para fines de análisis de datos, en cada tema 
que se pretende estudiar se utiliza una puntuación para medir cada respuesta, teniendo en cuenta las diferentes alternativas de selección, la medición se hará por cada sujeto que participe

Posterior a la elaboración del instrumento de recolección de infornación, el mismo se sometió a validez para ser aplicado en la investigación. Para tal efecto, se estimó la validez de contenido, con la verificación de las áreas y contenidos medidos por el instrumento de estudio, a través de la opinión de expertos en el área. El instrumento se sometió a un proceso de validación del contenido a través del juicio de diez (10) expertos. En otro orden de ideas, la confiabilidad, se ejecutó aplicando una prueba piloto, lo cual permitió determinar el índice de confiabilidad para su aplicación posterior a las unidades poblacionales del estudio, alcanzando un índice de $\alpha=$ 0,91 .

En consecuencia, para dar sentido a la información recolectada, se hizo uso de la estadística descriptiva, para describir, analizar y representar un los datos, utilizando métodos numéricos y tablas, en virtud de que una vez obtenidos en la aplicación del instrumento, se proceda a su codificación y tabulación con el programa SPSS versión XXIII.

Consecutivamente se procedió a la estimación de las medidas de tendencia central (media y moda), así como las medidas de dispersión (desviación estándar, valor mínimo y valor máximo) para determinar el comportamiento de la variable, estimando frecuencias absolutas ( $\mathrm{fa}$ ) y relativas ( $\mathrm{fr}$ ), con el fin de determinar la representatividad de las medias obtenidas.

Como se refirió en párrafos anteriores, fue necesario calcular las medidas de rango y desviación estándar en función de detallar la dispersión de la muestra en la escala de de medición, considerando el rango la extensión total de los datos en la escala. En lo que respecta a la desviación estándar, se concibió como el promedio de las desvicaciones de las puntuaciones con respecto a la media de la medición de distribución. de las respuesta de los participantes.

Este último puede determinar su intervalo, categoría y descripción, como se muestra en el Cuadro №3, lo que ayuda a explicar los resultados de la investigación y así poder inferir los aspectos de interés relacionados con la investigación. Al respecto, Hernández et al. (2017) expresan que la medida de variabilidad es un intervalo, que representa la dispersión de los datos dentro del rango de medición, siendo los más utilizados el rango, la desviación estándar y la varianza.

Cuadro 3

Baremo para la desviación estándar

\begin{tabular}{|c|c|c|l|}
\hline Rango & Intervalo & Categoría & \multicolumn{1}{|c|}{ Descripción } \\
\hline 1 & $4-5,00$ & $\begin{array}{c}\text { Muy alta } \\
\text { dispersión }\end{array}$ & $\begin{array}{l}\text { Muy alto nivel de dispersión de las respuestas y una muy baja } \\
\text { confiabilidad de las mismas. }\end{array}$ \\
\hline 2 & $3-3,99$ & Alta dispersión & $\begin{array}{l}\text { Alto nivel de dispersión de las respuestas y una muy baja } \\
\text { confiabilidad de las mismas. }\end{array}$ \\
\hline 3 & $2-2,99$ & $\begin{array}{c}\text { Moderada } \\
\text { dispersión }\end{array}$ & $\begin{array}{l}\text { Moderado nivel de dispersión de las respuestas y una baja } \\
\text { confiabilidad de las mismas. }\end{array}$ \\
\hline 4 & $1-1,99$ & Baja dispersión & $\begin{array}{l}\text { Bajo nivel de dispersión en las respuestas y una alta confiabilidad } \\
\text { de las mismas. }\end{array}$ \\
\hline 5 & $0-0,99$ & $\begin{array}{l}\text { Muy baja } \\
\text { dispersión }\end{array}$ & $\begin{array}{l}\text { Un muy bajo nivel de dispersión en las respuestas y una muy alta } \\
\text { confiabilidad de las mismas. }\end{array}$ \\
\hline
\end{tabular}

Fuente: Elaboración propia (2019) 


\section{Resultados}

Tabla 1

Creatividad

\begin{tabular}{|c|c|c|c|c|c|c|c|}
\hline Estadística & & Nivel & Baremo & Respuesta & $\mathrm{Fa}$ & Prop & $\%$ \\
\hline Media aritmética & 4,5 & Muy alto & $4-5,00$ & $\begin{array}{c}\text { Completamente de } \\
\text { acuerdo }\end{array}$ & 24 & 0,46 & 46 \\
\hline Mediana & 3,8 & Alto & $3-3,99$ & Medianamente de acuerdo & 30 & 0,54 & 54 \\
\hline Moda & 4,7 & Moderado & $2-2,99$ & $\begin{array}{c}\text { Ni de acuerdo ni en } \\
\text { desacuerdo }\end{array}$ & 00 & 0,00 & 00 \\
\hline \multirow[t]{3}{*}{ Desv. Estándar } & 0,6 & Bajo & $1-1,99$ & $\begin{array}{l}\text { Medianamente en } \\
\text { desacuerdo }\end{array}$ & 00 & 0,00 & 00 \\
\hline & & Muy bajo & $0-0,99$ & $\begin{array}{c}\text { Completamente en } \\
\text { desacuerdo }\end{array}$ & 00 & 0,00 & 00 \\
\hline & & & $\mathrm{N}^{\circ}$ ítemes 15 & $\mathrm{~N}^{\circ}$ sujetos 54 & 54 & & 100 \\
\hline
\end{tabular}

Fuente: Elaboración propia (2019)

En cuanto al aspecto central de la creatividad, como parte del perfil del emprendedor, principalmente se obtuvo que existe una marcada tendencia hacia las respuestas positivas, es decir, el $100 \%$ de las opiniones se distribuyeron entre las alternativas Completamente de Acuerdo (CA) y Medianamente de Acuerdo (MA).

Por otro lado, al analizar los promedios alcanzados por el indicador e insertarlo en el baremo, se evidencia un nivel muy alto para la media aritmética con 4,5; la mediana 3,8 y la moda alcanzó un valor de 4,7. Al considerar la desviación estándar como medida de dispersión, la cual fue de 0,6, se encontró que el indicador creatividad no cambió de posición con respecto a la dimensión.

De lo antes explicado, se establece que los docentes consideran que los estudiantes son creativos, pues cuentan con la habilidad de desarrollar, proponer y ejecutar decisiones en función de desarrollo de su potencialidad, en virtud de dar respuesta y adaptarse a los cambios y exigencias del entorno social.

Siguiendo a Bellon (1998), el proceso creativo se divide en varias etapas: preparación (acumulación de materiales, involucrando trabajo similar, adquisición de ideas, conocimientos y materiales), incubación (esta es una etapa inconsciente, una espera etapa). Los materiales acumulados deben asentarse), iluminación (aparece la inspiración, este es el momento en que estalla el comportamiento creativo); formulación (organización del pensamiento en sentido lógico); verificación (una autocrítica final en la que se revisa el valor del producto o descubrimiento).

Estos resultados coinciden con Jaimes (2009), donde involucra que un emprendedor busca desarrollar la creatividad y son aquellos, que logran alcanzar sus objetivos a través de la habilidad para influir, guiar, motivar e integrar los esfuerzos de otros. Desde esa perspectiva, se logrará la gestión con grupos de individuos, con finalidades comunes, compromiso y responsabilidad adquirida, para lo cual, deben poseer habilidades para influir en los entes involucrados, en este sentido, todos los participantes se beneficiarían de este proceso, desarrollando perspectivas más amplias, aceptando desarrollar competencia en un nivel más alto. 
Gráfico № 2

Acciones creativas

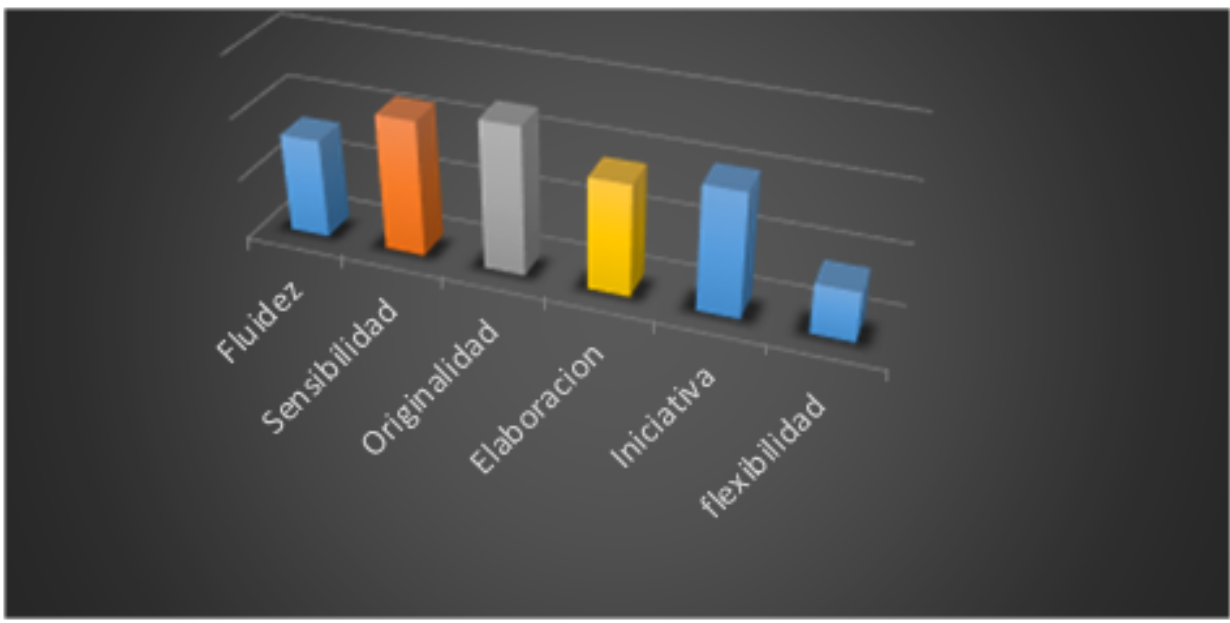

Fuente: Elaboración propia (2019)

El gráfico permite evidenciar como los docentes perciben los elementos de las acciones creativas en los estudiantes universitarios, en un matiz de influencia de unos sobre otros. Se puede indicar, que el espíritu creativo es entendido como una forma de pensar, razonar y actuar centrada en las oportunidades, planteadas con visión global y llevada a cabo mediante un liderazgo equilibrado y la gestión de un riesgo calculado.

Por otro lado, la creatividad está ligada a la habilidad de aprovechar las capacidades individuales para visualizar los problemas existentes como posibles oportunidades de mejora. Para ello, el gerente para cumplir de forma eficiente con sus funciones debe tener una amplia visión de las fortalezas y debilidades de cada uno de los integrantes de la organización, con el fin de aprovecharlas en la transformación de los problemas presentados en potenciales oportunidades.

En la actualidad, la creatividad se considera parte integrante del fomento y promoción del trabajo educativo en todos los campos. En la literatura relacionada se observa la conceptualización de la creatividad, lograr la división entre fomento y promoción no parte de la practica, mas bien, con un propósito académico de definición conceptual. En realidad, se podría creer los dos aspectos como continuos e interdependientes: si se genera un producto este procedería del proceso que lo crea, asu vez, este proceso depende de la capacidad y potencialidades humana, y el desempeño del factor humano tiene dependencia del entorno en el que se suscribe. Los cuatro elementos presentados configuran el proceso continuo de relaciones dialécticas a los cuales todo individuo se ve avocado.

\section{Conclusiones}

Se evidencia que los docentes están orientados a detectar las acciones que el estudiante ejecuta en función de desarrollar su perfil emprendedor, destacando que estos son creativos, cuentan con la habilidad de desarrollar, proponer y ejecutar decisiones en función del desarrollo de procesos y de propuestas empresariales, adaptándose a los cambios y exigencias del entorno social.

En términos generales, estos resultados comparados a los obtenidos por Niebles et al., (2018) demuestran un alto cumplimiento de actividades y procesos que la institución considera pertinente para corroborar el perfil de un emprendedor, relacionados con la flexibilidad, la fluidez, la originalidad entre otros, destacando aspectos relacionados con el nivel técnico del proceso creativo como parte del proceso educativo. 


\section{Referencias bibliográficas}

Aristimuño, M., \& Guaita, W. (2011). Las competencias gerenciales en la gestión de instituciones de educación superior. Posgrado y Sociedad. Revista Electrónica del Sistema de Estudios de Posgrado, 11(2), 63-76

Bellón, F. (1998). Descubrir la creatividad: desaprender para volver a aprender. Anaya-España.

Camero, V. (2008). Paradigma innovador de Liderazgo. Compilación. Madrid - España. Editorial Mc Graw Hill.

Connor, A. (2009). Impact of entrepreneurship education. Kansas City: Kauffman Center for Entrepreneurial Leadership.

Corbos, R. A., Zamfir, A., \& Florea, A. I. (2013). Strategic Managerial Implications of Supplier Segmentation in the Current Competitive Environment. In Proceedings of the 7th International Management Conference" New Management for the New Economy (pp. 359-365).

Durán, S. Parra, M. \& Márceles, V. (2015). Potenciación de habilidades para el desarrollo de emprendedores exitosos en el contexto universitario. Revista Opción, 77, 200-215

García, J. Durán, S., Parra, M. \& Martínez, H. (2018). Inserción, integración y equidad en el ámbito laboral: Escenario empresarial posconflicto en Colombia.

Gómez Ortiz, R. A. (2008). El liderazgo empresarial para la innovación tecnológica en las micro, pequeñas y medianas empresas. Pensamiento \& gestión, (24), 157-194.

Guilford, J. (1977). Will the real factor of extraversion-introversion please stand up? A reply to Eysenck.

Hernández, Y., \& Hernández, M. (2008). Nuevo rol del docente del siglo XXI. Recuperado el, 23

Hernández, H. Martínez, D. \& Rodríguez, J. (2017). Gestión de la calidad aplicada en el mejoramiento del sector universitario. Revista Espacios, 38(20).

Hernández, I. Parra, M. García, J. Castro, N. Romero, C. \& Durán, S. (2018). Comportamiento Organizacional Ciudadano (COC) como ejemplo de participación generador de un diálogo de saberes. Revista Espacios, 39(7)

Jaimes, V. (2009). Talento Humano mediante Competencias. Bogotá - Colombia. Grupo Editorial Norma

Jiménez, A. Hernández, H. \& Pitre, R. (2018). Social entrepreneurship and their impact on economic development from inclusive businesses. Logos ciencia \& tecnología, 10(2), 198-211.

Jiménez Leube, J. (2015). Perfil del emprendedor y fomento del emprendimiento: un enfoque personalista. Revista de Estudios de Juventud, (107), 165-180.

Moreno, M. Tezón, M. Rivera, T. Durán S. \& Parra M. (2018). Autoestima: Desarrollo de la autonomía personal en estudiantes del área técnica. Revista Espacios, 39(46)

Niebles, L., Niebles, W., Rodero, A., Barrios, I., \& Hernández, H., (2018). Management Skills and Leadership Styles: A Reflection with a Global Focus. Contemporary Engineering Sciences, Vol. 11, 2018, no. 54, 2661 2668

Niebles, W., Rodero, A., Niebles, L., Barrios, I., \& Hernández, H., (2018). Entrepreneurship and the Informal Economy: Characterization of Business in Colombia, an Analysis from the Global Entrepreneurship Monitor. Contemporary Engineering Sciences, Vol. 11, 2018, no. 54, 2651 - 2659 
Parra, M. Visbal, O. Durán, S. Badde, G. (2019). Calidad de la comunicación y actitud de los empleados ante procesos de cambio organizacional Interdisciplinaria, doi: https://doi.org/10.16888/interd.2019.36.1.11, Centro Interamericano de Investigaciones Psicológicas y Ciencias Afines, 36 (1), 155-170

Pérez, C. Tafur, J. Molina, G. \& Hernández, H. (2019). Análisis del Papel del Educador en el Postconflicto. Un Acercamiento desde la Percepción de la Ciudad de Barranquilla, en Colombia. Información tecnológica, 30(4), 199-208. https://dx.doi.org/10.4067/S0718-07642019000400199.

Pitre-Redondo, R., Rodriguez-Lopez, J., Hernández-Palma, H. G., \& Cardona-Arbelaez, D. (2017). Emprendimiento competitivo y productivo como renovador del sector salud en la Region Caribe. Revista Espacios, Vol 38, N 42, pag 7.

Pitre, R. Cardona, D. \& Hernández, H. (2017). Proyección del emprendimiento indígena como mecanismo de competitividad en el postconflicto colombiano. Revista de investigación, desarrollo e innovación, 7(2), 231-240.

Rebollo, C., \& Soubirón, E. (2010). La creatividad docente como factor generador de nuevos entornos de aprendizaje en la educación media. Buenos Aires: $n r$.

Rivera, J. Lay, N. Moreno, M. Pérez, A. Rocha, G. Parra, M. Durán, S. \& Redondo, O. (2019). Programa de entrenamiento para desarrollar habilidades sociales en estudiantes universitarios, Revista espacios, 40 (31), 13

Rivera, R. \& Santos, D. (2015). El perfil de los futuros emprendedores sociales: competencias y estilos de vida. Revista de Estudios de Juventud.

Sánchez, M. (2008). La medición del impacto de la ciencia y la tecnología en el desarrollo social. Ponencia en el Taller de Indicadores de Impacto de la Ciencia y la Tecnología en el Desarrollo social, organizado por RICYT, Mar del Plata, Argentina

Sánchez, M. García, J. Steffen, E. \& Hernández, H. (2019). Estrategias Pedagógicas en Procesos de Enseñanza y Aprendizaje en la Educación Superior incluyendo Tecnologías de la Información y las Comunicaciones. Información tecnológica, 30(3), 277-286. Doi: https://dx.doi.org/10.4067/S0718-07642019000300277

Valencia, A. Montoya, I. \& Montoya A. (2016). Intención emprendedora en estudiantes universitarios: Un estudio bibliométrico. Intangible Capital, 12(4), 881-922.

Viloria, A. Rodríguez, J. Payares, K. Vargas, C. Duran, S. Hernández, H. \& Arrozola, M. (2019). Determinating Student Interactions in a Virtual Learning Environment Using Data Mining, doi: https://doi.org/10.1016/j.procs.2019.08.082, Procedia Computer Science, 155, 587-592

Viloria Silva, A. Crissien Borrero, T. Vargas Villa, J. Torres Samuel, M. Garcia Guiliany, J. Vargas Mercado, C. Orellano Llinas, N. Batista Zea, K. (2019). Differential evolution clustering and data mining for determining learning routes in Moodle. https://doi. org/10.1007/978-981-32-9563-6_18.

Williams, A., Ostwald, M., \& Askland, H. (2010). Assessing creativity in the context of architectural design education. Proceedings of DRS. Attribución-NoCommercial 4.0 International

$(\mathrm{Cc}) \mathbf{B Y}$-NC 